\title{
Gestión del conocimiento y desafíos en PyMEs proveedoras de la cadena de hidrocarburos en la Cuenca Neuquina. Avance de investigación y reflexión para el diseño de políticas
}

\author{
Susana Graciela Landriscini \\ Universidad Nacional del Comahue \\ Lisandro Valentin Robles \\ Universidad Nacional del Comahue
}

\section{Resumen}

El documento aborda un conjunto de aspectos que destacan en el desenvolvimiento de las PyMEs de hidrocarburos de la Cuenca Neuquina, en la Norpatagonia argentina. La indagación está motivada, por un lado, en los cambios recientes del sector energético en general, e hidrocarburífero en particular, la caída de los precios del petróleo y una tendencia hacia energías más limpias. Y por otro, a nivel estructural, por la dinámica de innovación que se ha abierto con el desarrollo de los reservorios no convencionales para las firmas del segmento PYME, que integran las cadenas globales de valor del gas y el petróleo. La nueva fase de desarrollo de los hidrocarburos exige nuevos sistemas técnicos en la perforación y terminación de pozos y nuevos procedimientos en la extracción, así como cambios en los insumos, componentes y equipos. Ellos movilizan una nueva organización del trabajo, la introducción creciente de las TICS, la toma de información ad hoc, capacitación específica, y una rigurosa coordinación de procesos que se traduce en una nueva división del trabajo aguas arriba. En este marco, desde un enfoque evolucionista de la economía, se analizan un conjunto de problemáticas que enfrentan grupos de PyMEs regionales proveedoras de bienes y servicios del rubro electrónica y metalmecánica. Se indagó acerca de las rutinas de las firmas en la construcción de capacidades tecnológicas, el desarrollo de nuevos procesos y productos, los vínculos externos y las mejoras de gestión internas, los cambios en la productividad, y la competencia comercial en el nuevo escenario de volatilidad de los precios del petróleo.

Palabras clave: PyMEs, reservorios no convencionales de hidrocarburos, nuevos sistemas técnicos 


\title{
Knowledge management and challenges in SMEs supplying the hydrocarbon chain in the Neuquén Basin. Advancing research and reflection for policy design
}

\begin{abstract}
Sumary
The document refers to a set of aspects that stand out in the development of the small and medium enterprises of hydrocarbons in the Neuquén Basin, in the Argentine Norpatagonia. The investigation is motivated, on the one hand, by recent changes in the energy sector in general, and hydrocarbons in particular, the fall in oil prices and a trend towards cleaner energies. And on the other hand, at a structural level, due to the dynamics of innovation that has opened up with the development of unconventional reservoirs for companies in the SME segment, which make up the global value chains for gas and oil. The new development phase of hydrocarbons requires new technical systems in the drilling and completion of wells and new extraction procedures, as well as changes in inputs, components and equipment. They mobilize a new organization of work, the increasing introduction of ICTs, the taking of ad hoc information, specific training, and a rigorous coordination that generated a new division of work upstream. In this framework, from an evolutionary approach to the economy, a set of problems faced by groups of regional SMEs that provide goods and services from the electronic and metal-mechanical sector are analyzed. It inquired about the routines of firms in the construction of technological capabilities, the development of new processes and products, external links and internal management improvements, changes in productivity, and commercial competition in the new scenario of volatility of oil prices.
\end{abstract}

Keywords: SMEs, unconventional hydrocarbon reservoirs, new technical systems

\section{Gerenciamento de conhecimento e desafios em fornecedores de PME da cadeia de hidrocarbonetos na bacia de Neuquén. Avançando pesquisa e reflexão para o desenho de políticas}

\section{Resumo}

O documento aborda um conjunto de aspectos que se destacam no desenvolvimento das PyMEs de hidrocarbonetos na Bacia do Neuquén, na Norpatonia da Argentina. A investigação está motivada, por um lado, pelas mudanças recentes no setor de energia em geral, e os hidrocarbonetos em particular, a queda nos preços do petróleo e uma tendência para energias mais limpas. E, por outro lado, a nível estrutural, devido à dinâmica da inovação que se abriu com o desenvolvimento de reservatórios não convencionais para empresas do segmento de PMEs, que compõem as cadeias de valor globais de gás e petróleo. A nova fase de desenvolvimento de hidrocarbonetos requer novos sistemas técnicos na perfuração e conclusão de poços e novos procedimentos de extração, bem como mudanças em insumos, componentes e equipamentos. Eles mobilizam uma nova organização de trabalho, a introdução crescente de TICs, a tomada de informações ad hoc, treinamento específico e uma coordenação de processo rigorosa que se traduz em uma nova divisão de trabalho no upstream. Neste contexto, a partir de uma abordagem evolutiva para a economia, um conjunto de problemas enfrentados pelos grupos de PME regionais que fornecem bens e serviços do setor eletrônico e metal mecânico são analisados. Preguntou sobre as rotinas das empresas na construção de capacidades tecnológicas, o desenvolvimento de novos processos e produtos, links externos e melhorias na gestão interna, mudanças na produtividade e concorrência comercial no novo cenário de volatilidade dos preços do petróleo.

Palavras-chave: PMEs, reservatórios de hidrocarbonetos não convencionais, novos sistemas técnicos 


\section{INTRODUCCIÓN}

El presente trabajo aborda aspectos de la gestión del conocimiento y la organización del trabajo en proveedoras regionales PyMEs del complejo de hidrocarburos en la Cuenca Neuquina, Norpatagonia Argentina. Está motivado por los cambios que evidencia al presente el sector hidrocarburífero a nivel mundial y nacional (FUNSEAM, 2015), que imponen crecientes niveles de eficiencia y competencia; y en lo estructural, por el reconocimiento de las restricciones estratégicas y operativas registradas en dichas firmas., que demandan la implementación de mejora técnico productivas y de gestión para su supervivencia y consolidación como parte del segundo anillo de proveedores de las operadoras en la cadena de los hidrocarburos, y/o su ascenso en la misma.

De la reciente literatura en temas de innovación, se obtienen trabajos de autores neo y post schumpeterianos y neoestructuralistas que dan cuenta de las oportunidades que se abren en las actividades y economías con base en los recursos naturales, asociadas a las nuevas tecnologías (Marín, 2017; Pérez, 2016) y a los procesos de aprendizaje y desarrollo de competencias en las firmas más allá de los laboratorios de I\&D, sea a partir de la generación de mejoras en productos y procesos, en organización, y/o en las formas de llegar al mercado (Barletta, Pereira, Suárez y Yoguel, 2016, Yoguel, Barletta y Pereira, 2013; ) 1 Aggio, Lengyel, Milesi y Pandolfo (2016) lo plantean específicamente para los desarrollos de reservorios de hidrocarburos no convencionales, que marcan una nueva etapa de expansión de la frontera productiva en la economía mundial y en Argentina. Es sí que la nueva fase de desarrollo de los reservorios no convencionales contenidos en la formación geológica Vaca Muerta en la Cuenca Neuquina, exige nuevos sistemas técnicos asociados a la perforación y terminación de pozos en la exploración y la extracción, cambios en procesos y el uso de insumos, y la introducción de ajustes y cambios en los componentes y equipos, que plantean la necesidad de una nueva organización del trabajo, toma de información ad hoc, capacitación específica y una rigurosa coordinación de procesos y división del trabajo en el upstream.

En este marco, a partir de un Proyecto de Vinculación Tecnológica, desarrollado por los autores en la Universidad Nacional del Comahue, Neuquén, en 2015/2016, de modo asociado a técnicos del Instituto Nacional de Tecnología Industrial, sede Neuquén, y del Programa Sustenta de Desarrollo de Proveedores de la compañía hidrocarburífera nacional YPF, se avanzó en el análisis de un conjunto de problemáticas que enfrentan firmas del segmento PyME que proveen servicios y bienes del rubro electrónica y metalmecánica. Se abordaron, articulando la teoría, la dinámica tecnológica de la actividad, y un relevamiento de campo orientado a casos de proveedoras del rubro, las rutinas de las firmas en la construcción de
1 La recuperación de las ideas de Schumpeter acerca de la destrucción creativa en los procesos de desenvolvimiento económico, dio lugar a la generación de una nutrida tradición de trabajos evolucionistas y neoschumpeterianos que produjeron un renacimiento y actualización del pensamiento del autor, desarrollando a su vez nuevas dimensiones analíticas ausentes en su obra. Siguiendo a Yoguel et al, 2013, pueden citarse algunos autores que desde distintas perspectivas se pueden encuadrar en esta corriente. Pueden citarse, entre otros, Antonelli, 2011; Dopfer, 2006; Dosi, 2000; Fagerberg, 2003;

Freeman, 1995; Foster, 2000; Langlois, 2007; Lundvall, 1992; Malerba y Orsenigo, 1997; Metcalfe, 1998, 2010; Perez, 2010; Reinert, 2000; Rosemberg, 2011; Saviotti, 1996; Witt, 2002. 
capacidades tecnológicas y en el desarrollo de nuevos procesos y productos; los vínculos externos, las mejoras de gestión internas, y la competencia por la captación de clientes en el nuevo escenario de bajos del petróleo a nivel mundial, y de orientación al gas.

\section{Referencias teóricas que guiaron la investigación: PyMEs e innovación}

La temática se vincula a los procesos de innovación y el cambio tecnológico en organizaciones. En función de ello, y reconociendo que los enfoques ortodoxos de las firmas no dan respuesta al pretender explicar los problemas frecuentes de las mismas cuando operan en tramas jerárquicas internacionalizadas, en las que enfrentan asimetrías informativas y barreras a la entrada, se ha recurrido a los aportes de la Economía Evolucionista Neoschumpeteriana, que incorporan la cuestión de la trayectoria de las empresas, la de sus titulares y la de los planteles de trabajadores en el análisis de los procesos de transformación (Yoguel, et al, 2013; Barletta, et al, 2014; Barletta et al, 2016). Agregado a ello, se han consultado los enfoques sobre la gestión de la información y la organización del trabajo que plantean la importancia de las formas horizontales y participativas de construcción del conocimiento y la toma de decisiones en las firmas, en contraste con los modos tayloristas de organización (Erbes et al, 2014). Se han retomado, asimismo, elementos de la Economía Institucional referidos a las relaciones entre firmas, las aglomeraciones en el territorio y los vínculos con el entorno científico y las cadenas de valor. Se ha recurrido a tal fin a los desarrollos sobre aglomeraciones, sistemas de innovación y economías de localización; cadenas de valor, y procesos de upgrading. (Antonelli, 2011; Becattini, 2002; Dosi, 2000; Kaplinsky, 2012; Lundvall, 2012; Pérez, 2016; Pietrobelli y Rabellotti, 2005; entre otros).

El enfoque económico evolucionista se aparta del análisis estático ortodoxo, en tanto busca identificar las reglas que rigen la transformación económica, teniendo en cuenta la complejidad en las interacciones de las dinámicas micro, meso y macroeconómicas. Siguiendo a Schumpeter, "La innovación y el cambio tecnológico son considerados factores claves para explicar el crecimiento y la transformación económica" (Barletta, et al , 2014: 11). En cuanto a los comportamientos de las firmas, esta corriente identifica tres tipos de rutinas: (i) operativas; (ii) de modificación y (iii) de búsqueda de alternativas para introducir mejoras y desarrollar nuevas ventajas competitivas. De ello se deriva el concepto de capacidades dinámicas de las firmas. La relación entre los conceptos de capacidades tecnológicas y gestión del conocimiento es evidente si se considera que "la capacidad de crear, integrar, transferir y utilizar conocimiento en una forma continua apuntala las capacidades de la firma y sus ventajas competitivas" (Easterby-Smith y Prieto, 2007: 33). Adicionalmente, existe cierto consenso en la literatura heterodoxa acerca del rol de las PyMEs en la economía, especialmente a partir de los estudios de los Distritos Industriales Italianos (Becattini, 2002; Dini y Stumpo, 2011; Ferraro y Stumpo, 2010) y de los Clusters (Porter, 1992), la valorización de la flexibilidad (Teece y Pisano, 1994; Torres, 2004), y su impacto en el empleo y la innovación (Kantis, et al 2011).

A partir de lo anterior, resulta de interés explorar las estrategias de gestión del conocimiento en PyMEs proveedoras en la Cuenca Hidrocarburífera Neuquina, lo 
que se asocia a la organización del trabajo, el aprendizaje, los vínculos con el entorno y el papel del dueño gerente como elemento central en la gestión de la firma, a fin de conocer los factores que inducen al éxito, y los que retrasan la obtención de mejoras en la perfomance de las empresas, en su participación en los mercados, y en la relación con los clientes de la cadena de valor.

El problema de la gestión del conocimiento resulta un desafío particularmente relevante en la industria de los hidrocarburos, reconocida hasta no hace mucho tiempo como una industria madura, que al presente evidencia novedades resultantes de la exploración y explotación de reservorios no convencionales que se retroalimentan con una cantidad creciente de conocimientos aplicados al proceso productivo, tanto de forma incorporada como desincorporada. Ello abre nuevas oportunidades. En este marco, adquieren creciente relevancia las problemáticas vinculadas a la generación y retención de los conocimientos ante una tendencia al envejecimiento y la disminución del total de trabajadores, y la complejidad del proceso productivo en la explotación de yacimientos no convencionales (Di Sbroiavacca, 2013); la intensidad en el uso de conocimiento de esta industria en particular, la necesidad constante de reducción de costos operativos y el incremento de la eficiencia frente a la volatilidad de los precios internacionales (Landriscini y Carignano, 2016).

En el caso de las proveedoras PyMEs regionales insertas en distintas tramas empresarias en los hidrocarburos, la necesidad que experimentan de generar nuevo conocimiento, y de almacenarlo para su reutilización, proviene tanto de sus propias operaciones como también de las exigencias de sus clientes, empresas nacionales y extranjeras con las que se hallan relacionadas, por lo general con débiles posiciones de negociación (Landriscini, Robles y Carignano, 2015 ). En ese marco, Landriscini y Orlandini (2014) reflexionan en torno a las "formas de co creación y difusión de conocimientos en redes formales e informales, resultado de la aglomeración y la trama de relaciones". Además, tal como indican Neuman et al. (2012) más del 90\% de las empresas proveedoras de bienes y servicios en la industria de hidrocarburos, y que se relacionan particularmente con YPF, son PyMEs de capitales nacionales y evidencian problemas de escala, restricciones tecnológicas y comportamientos idiosincráticos.

Agregado a ello, cabe señalar qué se entiende por innovación a los efectos del presente trabajo, y evaluar la posibilidad que tienen este segmento de empresas que operan en la Cuenca Neuquina, de desarrollar esfuerzos de innovación, de introducir mejoras en procesos, productos y gestión, y en los vínculos con las instituciones de I\&D. La dinámica económica se desenvuelve entre la inercia y la innovación a nivel micro, sujeta a validación en el entorno del mercado y las instituciones. Cabe preguntarse entonces: qué oportunidades y desafíos encuentran las PyMES en este proceso de competencia evolutiva? La teoría ofrece respuestas diversas, que pueden inspirar distintos interrogantes para explorar junto a los responsables de empresas, y otras provienen de experiencias comparadas desarrolladas en particulares momentos históricos, entornos institucionales, niveles de desarrollo y tamaño de las economías, y que corresponden a distintas ramas de actividad y modos de organización del trabajo y de gestión del conocimiento en las firmas. 
A partir de la década de 1990, una serie de contribuciones inspiradas en el análisis de los aglomerados de empresas, sobre todo en las experiencias de los distritos industriales italianos (Pyke, Becattini y Sengenberger 1990; Pyke y Sengenberger 1992; Nadvi 1995; Humphrey y Schmitz 1996; Dossi, 1998), impulsaron el desarrollo de un enfoque sistémico de la competitividad que interpreta la innovación como un proceso de aprendizaje. En dicho proceso, otorgan particular peso a la trayectoria de los agentes (path dependence) en la que resulta determinante la interacción entre la empresa y su entorno productivo e institucional (Lundvall, 2005, 1985; Nelson \& Winter, 1982). Esta interpretación reconoce, por un lado, que no existe una sola vía óptima para la generación de innovaciones $\mathrm{y}$, por otro, que la interpretación tradicional que relaciona de manera lineal la generación de innovaciones con las actividades de I+D, es una simplificación extrema de un proceso mucho más complejo (Dini y Stumpo, 2011; Barleta et al, 2016; Yoguel, et al, 2013).

De lo anterior, se desprenden dos consecuencias importantes: 1. Se adopta en el trabajo la definición amplia de innovación que presenta la tercera versión del Manual de Oslo, considerando como esfuerzos innovadores a las mejoras organizativas y de gestión agregadas a las productivas y no sólo los resultados ${ }^{2}$; y 2 . Se indaga acerca de la eficacia de las estrategias informales de innovación, las que dependen del contexto en que operan las PyMEs. En sectores en que las estrategias competitivas predominantes hacen un uso intensivo de investigaciones de base para el desarrollo de un proceso permanente de diversificación de los escenarios cognitivos y tecnológicos de referencia, las posibilidades competitivas de las PyMEs se reducen. Por el contrario, en contextos en los que la competitividad se basa en una capacidad de adaptación rápida de productos, procesos u organización, en un escenario caracterizado por una base cognitiva relativamente estable, las estrategias informales de innovación pueden tener éxito (Dini y Stumpo, 2011; Katz y Zamorano, 2011).

La importancia del entorno en el que las firmas se desempeñan no sólo está relacionada al sector económico en que actúan, sino también al contexto empresarial-institucional. En este sentido, los sistemas productivos y de innovación locales pueden influir ya sea generando capacidades colectivas de análisis de las tendencias de los mercados y escenarios competitivos, y brindando -o no- estímulos para la apertura de los agentes para relacionarse externamente y para su interés en la observación y la búsqueda de nuevas realidades y nuevas perspectivas. Eso depende tanto de estrategias colectivas explícitas que contemplen estas metas, como de valores y tradiciones acumuladas que caracterizan la cultura productiva y social de la comunidad considerada. Además, una serie de características de la comunidad local influencian las posibilidades de respuesta de parte de los actores locales. La capacidad cognitiva/innovativa de los mismos
2 En el citado Manual, se define la innovación como la concepción e implantación de cambios significativos en el producto, el proceso, el marketing o

la organización de la empresa con el propósito de mejorar los resultados. Los cambios innovadores se realizan mediante la aplicación de nuevos conocimientos y tecnología que pueden ser desarrollados internamente, en colaboración externa o adquiridos mediante servicios de

asesoramiento o por compra de tecnología.

Las actividades de

innovación incluyen

todas las actuaciones científicas, tecnológicas, organizativas, financieras y comerciales que conducen a la innovación. Se consideran tanto las que hayan producido éxito, como las que estén en curso o las realizadas dentro de proyectos cancelados por falta de viabilidad. La innovación implica la utilización de un nuevo conocimiento o de una nueva combinación de conocimientos existentes. El nuevo conocimiento puede obtenerse por la vía de I+D (como una de las etapas del proceso de innovación) y de Otras actividades innovadoras

(que no son $\mathrm{I}+\mathrm{D}$ pero forman parte de la innovación) (Manual de Oslo; análisis de Sixto Jansa, Portal OTRIUNED, 2010, pp. 2-3). 
depende de manera significativa de las características del sistema productivo territorial considerado y de la división del trabajo alcanzada entre las empresas que lo conforman y su trayectoria. En la medida en que en un sistema local se generan externalidades y procesos cooperativos de aprendizaje, sus integrantes podrán contar con activos que acrecientan sus capacidades competitivas. Para integrar los conocimientos individuales con los del entorno, disminuir la incertidumbre estratégica (Kline y Rosenberg, 1986), y potenciar los procesos de aprendizaje, las empresas requieren un umbral mínimo de competencias que va aumentando a medida que los sistemas pierden virtuosidad" (Yoguel et al, 2005: 76). Por caso, las actividades en el sector hidrocarburos, en el que predominan las tramas empresarias jerárquicas en las cadenas de valor, plantean fuertes exigencias a las PyMEs en materia de actualización tecnológica, certificación, normas de procesos y auditoría de productos, y en cuanto a flexibilidad, costos y tiempos de respuesta. De este modo, cuanto mayor es la "proximidad cognitiva" tanto más efectiva resulta la observación y más fácil la movilidad de los recursos humanos al interior del sistema productivo. A ello Torre (2004) agrega la "proximidad geográfica, organizacional, social, e institucional". En cuanto a la capacidad de generación de conocimientos, los autores señalan que el análisis de los distritos industriales italianos indica que este proceso resulta tanto más intenso cuanto más desarrollada es la división externa del trabajo y más reducida es la división del trabajo al interior de las firmas.

En síntesis, la capacidad de un sistema productivo de absorber y difundir nuevos conocimientos tiene que ver con un conjunto de características de su estructura, entre las que destacan: el número de actores independientes que lo conforman; la intensidad de sus vínculos y de los mecanismos que facilitan su interacción; del modelo de división interna y externa del trabajo; y del nivel de proximidad cognitiva que se registra entre los integrantes del sistema considerado. (Dini y Stumpo, 2011)

En tal sentido, es muy relevante el papel de las universidades generando densidad institucional para la I\&D y transferencia tecnológica que coopere al desarrollo de innovaciones, mejoras de productividad y apropiación horizontal de resultados. En el caso de las cadenas de fuerte acoplamiento entre proveedores y clientes aparecen consideraciones particulares que se vinculan a lo tecnológico: la posición de las PyMEs en la cadena, la especificidad de los procesos y de los bienes y servicios, la circulación de información, la complementariedad, la asistencia postventa, y lo económico-financiero, ligado a la estructura del mercado, el grado de asimetría en las relaciones y las condiciones contractuales. También son numerosos los estudios que ampliando la unidad de análisis han adoptado una visión de tramas empresarias en cadenas globales de valor y de cluster. En ellos se ha puesto el foco en los temas de la coordinación de los actores públicos y privados y los relativos a la generación y gestión eficiente de bienes o servicios competitivos (Pietrobelli y Rabellotti, 2005) 
De acuerdo con Lall (1992), las capacidades tecnológicas a nivel de un país se ordenan en tres categorías: inversión física, capital humano y esfuerzos tecnológicos. El autor argumenta que las tres categorías son interdependientes, ya que si el capital físico se acumula sin las habilidades, o sin la tecnología necesaria para operarlo de modo eficiente, las capacidades tecnológicas nacionales no se desarrollarán de modo adecuado. Es decir que, la inversión física es una capacidad básica si la planta y el equipo son necesarios para que exista la industria, pero lo más importante es la eficiencia con la cual se utiliza el capital. El capital humano incluye no sólo las habilidades generadas por la educación y la capacitación formal, sino también las que se desarrollan con la práctica y la experiencia en las actividades y capacidades heredadas, que ayudan al desarrollo tecnológico. La calidad de la educación formal, y los programas de estudios con contenidos acorde a las nuevas necesidades tecnológicas revisten gran importancia. Así, la capacidad en esfuerzos tecnológicos se relaciona con la mano de obra especializada disponible para tareas técnicas, los gastos en I\&D formales, innovaciones, patentes, y otros indicadores de éxito tecnológico. Y el trabajo calificado y el capital físico son productivos sólo si se combinan con esfuerzos de las firmas para asimilar y mejorar la tecnología relevante. Es por ello que la acumulación de capacidades tecnológicas no constituye un proceso automático, inherente al libre funcionamiento de las leyes del mercado. Por el contrario, supone procesos arriesgados e imprevisibles. Al respecto Bell y Pavitt (1992) señalan tres etapas en los procesos de acumulación de capacidades tecnológicas: i) en la primera, la tecnología es adoptada para incorporarla en nuevas instalaciones; ii) en la segunda, se incrementa la eficiencia inicial y se modifica la tecnología adecuándose a los cambios en los mercados de insumos y productos; y iii) en la tercera, las empresas pueden basarse en las capacidades ya adquiridas para introducir un cambio técnico más sustancial, modificando los productos existentes; sustituyendo productos, diversificando los materiales de insumo y producto o modificando las tecnologías usadas. Este proceso de acumulación de capacidades parte de una base mínima de conocimientos tecnológicos, por la cual las empresas son tecnológicamente inmaduras, pues aprenden y acumulan conocimientos con el paso del tiempo; y permite encarar progresivamente nuevas actividades y adquirir capacidades tecnológicas, hasta que las empresas estén preparadas para llevar a cabo actividades de innovación (Ortega Rangel, 2005; Dutrénit, 2003, en Landriscini y Robles, 2016).

Existen así varias categorías de actividades tecnológicas en los países en desarrollo. Se ha identificado una secuencia en el proceso de adquisición o desarrollo de capacidad tecnológica, la que puede resumirse en las siguientes etapas: 1. Identificar una oportunidad o un problema; 2. Explorar las soluciones tecnológicas posibles, seleccionar las más apropiadas y, si es necesario, negociar la adquisición del nuevo conocimiento tecnológico; 3. Operar la tecnología; 4. Adaptar la tecnología a las condiciones locales; 5 . Modificar la tecnología en respuesta a los cambios del entorno económico; 6. Modificar sustancialmente el proceso o el producto; y 7 . Llevar a cabo I \& D internos de forma organizada y sistemática. Esta diferenciación resulta de gran utilidad para abordar los procesos de mejora, sus posibilidades y restricciones hacia adentro y en materia de vinculación con el entorno en las firmas PyMEs desafiadas por cambios técnico productivos en sectores maduros (como los hidrocarburos, y los asociados a ellos desde la 
metalmecánica y la electrónica), y por crecientes barreras a la entrada. (Ortega Rangel, 2005) Siguiendo esa literatura, la acumulación de capacidades tecnológicas de las empresas, aporta mejoras en la competitividad de las mismas, llevando a avanzar hacia una visión sistémica del concepto. En tal sentido, la corriente evolucionista señala como factor clave de la competitividad al factor tecnológico expresado en la capacidad de transformar insumos, incrementar la eficiencia en el uso de los mismos y crear productos y procesos de producción más complejos (Ortega Rangel, 2005).

\section{La gestión del conocimiento en las PyMEs: entre la teoría y la práctica}

Si bien podría considerarse que la gestión del conocimiento es un problema que deben resolver todas las firmas por igual, existen diferencias entre las empresas, las cuales afectan el punto de partida y los dispositivos que cada empresa puede utilizar para gestionar el conocimiento de forma exitosa. A los fines de la investigación, dado el foco puesto en las PyMEs, y que las mismas enfrentan algunos problemas de naturaleza diferente a los que aquejan a las empresas grandes, como fuera señalado se adoptó una posición más cercana a la heterodoxia que a la ortodoxia económica. Si bien existe abundante literatura que aborda la importancia de una gestión adecuada de los conocimientos en el contexto actual de capitalismo de innovación intensiva, ello no ocurre con referencia a la gestión de conocimiento en las PyMEs. A los efectos del estudio se retomó la revisión bibliográfica realizada por Durst y Edvardsson (2012) sobre gestión de conocimiento en PyMEs y el estudio de 25 casos que hacen Evangelista et al (2010) en la región del este de Nápoles. Se consultaron, asimismo, por su relevancia regional, el caso mexicano de la industria de manufacturas y el caso brasileño de la industria de hidrocarburos. Finalmente se retomó el trabajo de Erbes et al. (2014), titulado "El rol de la organización del trabajo en el desarrollo de procesos de aprendizaje", ya que desde la visión evolucionista neoschumpeteriana ofrece una herramienta analítica que es aplicable en las PyMEs para favorecer un manejo satisfactorio de los procesos de aprendizaje en las organizaciones.

Como es sabido, en el contexto actual el conocimiento se ha convertido en el factor estratégico más importante en la actividad empresarial, puesto que está asociado con las capacidades de las empresas para lograr una ventaja competitiva (Spender, 1996 y Teece, 2001 en Durst \& Edvardsson, 2012). Algunos estudios evidencian que hay una ausencia de gestión sistemática del conocimiento en las PyMEs, y si se implementa alguna medida, es menos sofisticada que las que se observan en grandes empresas (McAdam y Reid, 2001 y Wong y Aspinwall, 2005 en Durst \& Edvardsson, 2012). De la literatura sobre gestión de conocimiento en PyMEs se puede extraer en primera instancia una enumeración de las características más destacadas que por lo general se encuentran presentes en las empresas de menores dimensiones: i) limitaciones en el acceso a recursos, lo que trae aparejado que los recursos existentes deban ser utilizados con precaución (Jarillo, 1989 y Amelingmeyer y Amelingmeyer, 2005 en Durst \& Edvardsson, 2012); ii) estructura plana y orgánica, informal, no burocrática y con pocas reglas. La facultad de control suele estar concentrada en la supervisión personal por parte del dueño. iii) el dueñogerente suele tener un papel protagónico, concentrando con frecuencia los 
procesos de planificación y toma de decisiones y las funciones de control: así la operación diaria de una PyME exige tanta atención que deriva en que no se dedique suficiente tiempo a las cuestiones estratégicas. Esto sumado a la falta de recursos financieros y capacidades por lo general resulta en que el conocimiento termina siendo concentrado en la mente del empresario y algunos empleados clave en vez de ser almacenado físicamente o compartido (Durst y Edvardsson, 2012). iv) estas empresas suelen tener una menor escala de producción, pero gozan de mayor flexibilidad operativa; y v) entre ellas la heterogeneidad es la norma. Ello exige especial atención a la hora de hacer generalizaciones

La literatura reseñada por Durst y Edvardsson muestra una serie de desafíos comunes a todas las PyMEs en el campo de la gestión del conocimiento a las cuales se les puede agregar las peculiaridades mencionadas por Desouza y Awazu (2006 en Evangelista et al., 2010): por lo general, estas unidades no tienen una política explícita destinada a la gestión estratégica del conocimiento; de hacerlo, tratan a la cuestión desde el punto de vista operativo. Se focalizan más que las empresas grandes en el conocimiento tácito, ya que no suelen tener repositorios de conocimiento codificado. Dada la dependencia con respecto al conocimiento proveniente de fuentes externas, es más frecuente que los canales de comunicación existentes conecten empresas entre sí, en vez de que existan canales internos de transferencia de conocimiento.

Durst y Edvardsson (2012) identifican cinco actividades relacionadas a la gestión del conocimiento: la identificación, la creación o adquisición, el almacenamiento/retención, la transferencia y utilización, y estudian cuáles son los avances que ha habido en cada una de esas subdisciplinas. En cuanto a la identificación de los conocimientos que son relevantes para la firma, Durst y Wilhelm, 2011 (en Durst y Edvardsson, 2012) señalan la necesidad de hacer una distinción, al interior de la firma, entre el conocimiento que es fácilmente accesible o reproducible y aquél que es difícil de imitar. Con respecto a la creación de conocimiento o la adquisición de nuevo conocimiento de fuentes externas, la literatura indica que dadas las limitaciones propias de las PyMEs, las mismas se ven forzadas a recurrir predominantemente a fuentes externas. Si bien la mayoría de los estudios se enfocan en la forma en que se puede generar conocimiento al interior de la firma a través de conocimiento proveniente de fuentes externas, CegarraNavarro y Dewhurst (2006 en Durst y Edvardsson, 2012) prestan especial atención a la habilidad que la empresa debe tener para "desaprender" como precondición para incorporar nuevo conocimiento.

Otros estudios señalan el desconocimiento por parte de los empresarios PyME sobre estos temas, la escasa o nula inversión en I+D, la importancia de la implicación de las personas en la organización para la generación de nuevo conocimiento (Soon y Zainol, 2011, Pillarlia, 2008b, Ambrosini y Bowman, 2008, Hari, Egbu y Kumar, 2005 en Durst \& Edvardsson, 2012). Los estudios existentes sobre retención del conocimiento en PyMEs están marcados por la gran predominancia del conocimiento tácito en este tipo de empresas, así como también por el hecho de que el conocimiento suele estar concentrado en el dueño y unos pocos empleados claves. Al respecto, Durst y Gueldenberg, 2010 (en Durst y Edvardsson, 2012) señalan la necesidad de identificar y retener a los empleados que son depositarios del conocimiento vital para el funcionamiento de la firma. Dada la relevancia del 
conocimiento externo para las PyMEs, la transferencia inter-organizacional de conocimiento es crucial para este tipo de empresas. Sin embargo, resulta importante que el conocimiento no esté excesivamente concentrado en el dueño y en unos pocos empleados clave, lo cual exige que la transferencia interorganizacional se complemente con transferencia intra-organizacional. En dicho proceso entran en juego las capacidades de absorción que por lo general son una de las principales dificultades de las PyMEs. En cuanto a la utilización del conocimiento incorporado o desarrollado internamente, Durst y Edvardsson destacan la relevancia de esta actividad, puesto que es el objetivo que debe tener una estrategia de gestión del conocimiento: si el conocimiento generado o incorporado no se utiliza, entonces no se materializa en una ventaja competitiva. Los estudios hallados destacan la influencia que tienen en la utilización del conocimiento los objetivos estratégicos que establece el dueño de la PyME y la cultura que en consecuencia se genera al interior de la empresa.

Del análisis realizado por Evangelista et al. (2010), resulta que las herramientas de gestión de conocimiento más utilizadas por las PyMEs son grupos de trabajo, sitios de internet y el uso de una intranet, mientras que las menos utilizadas son los sistemas de apoyo a la toma de decisiones, data mining y sistemas de manejo de documentación (p. 39). Sin embargo, las empresas también han mostrado una serie de barreras para la implementación de herramientas de gestión del conocimiento, tales como la protección de información crítica, la falta de socios comerciales para compartir conocimiento y la cultura empresarial. "Resulta interesante que las barreras tecnológicas y la naturaleza tácita del conocimiento intercambiado son las barreras consideradas menos relevantes" (Evangelista et al. (2010) p. 40). A partir de ello, las recomendaciones que emergen del trabajo de Evangelista et al., consisten en generar mejores intercambios de conocimiento con los clientes para incrementar el conocimiento sobre mercados, tener en cuenta la gestión tecnológica a la hora de implementar una estrategia para gestionar los saberes, y no perder de vista el conocimiento relacional y la generación de una cultura que motive la colaboración entre los integrantes de la organización y la transferencia inter-organizacional de saberes.

\section{La organización del trabajo y la gestión del conocimiento}

Con respecto a la organización del trabajo, definida como los aspectos técnicos y sociales que intervienen en la producción de bienes y servicios, Erbes et al. (2014) consideran que los aspectos claves para dar cuenta del funcionamiento de la organización del trabajo son: por un lado, la "arquitectura" o diseño organizativo del trabajo, que incluye la comunicación y el intercambio de saberes, y, por otro, las herramientas de gestión que tengan el objetivo de "activar" dicha estructura en la práctica. Los autores consideran seis dimensiones vinculadas a las características que asumen las actividades productivas. A partir de las formas particulares que estas dimensiones adquieren, pero también en función de las interacciones existentes entre las mismas, surgen tres arquetipos organizacionales: la 
organización formativa en un extremo, la organización taylorista en el otro extremo, y la organización híbrida en el medio de ambas. ${ }^{3}$

Mientras en la organización taylorista el trabajo es individual, en la organización híbrida industrial el trabajo se lleva a cabo en equipo pero con limitada participación en la concepción del proceso, y en la organización híbrida de servicios el trabajo también es colectivo pero con relación indirecta con la producción. En la organización formativa, en cambio, se trabaja en equipo con amplia participación en la concepción del proceso. Agregado a ello, según Grimshaw et al., 2002 (en Erbes et al., 2014) la organización híbrida lleva a cabo programas de formación que abarcan a una amplia proporción de los trabajadores para el desarrollo de tareas repetitivas, con una escasa complejidad y gran generalidad de los temas considerados, con el objetivo de dotar a los empleados de habilidades que les permitan ocupar diferentes posiciones dentro de la organización; en cambio la organización formativa implementa programas sistemáticos apuntados a grupos específicos y centrados en temáticas relevantes para ellos. La "polivalencia enriquecedora" desarrollada al interior de las organizaciones formativas consiste en realizar diferentes tareas con niveles crecientes de complejidad y de las competencias requeridas para ejecutarlas. A su vez, la autonomía puede emerger como parte de un proceso dinámico y permitir la resolución de problemas, en cuyo caso resulta motivadora y fomenta los procesos de aprendizaje. La posibilidad de su desarrollo está sujeta a que el trabajador tenga la expertise necesaria para participar en las decisiones que atañen a su área, que los estilos de conducción sean más formativos que directivos y que el ambiente de trabajo físico y social estimule la interconsulta y la cooperación, con un bajo nivel de disciplinamiento de las interacciones. Mientras que en la organización taylorista la concepción y la ejecución están separadas, por lo que el trabajador no toma decisiones ante imprevistos en su trabajo, en la organización híbrida el trabajador interviene ante los imprevistos de la forma que se pautó previamente, y en la organización formativa el trabajador llega a organizar su propio proceso de trabajo. Respecto a los grados de participación en las decisiones, en la organización taylorista no existe ni fomento por parte de la empresa ni interés de parte de los trabajadores para participar, en la organización híbrida la participación es medianamente promovida por la empresa, y en la organización formativa confluyen el fomento de la participación por parte de la empresa y el interés de los trabajadores por participar. En cuanto a la flexibilidad, ella tiene que ver con la capacidad de los empleados de desarrollar diferentes tareas según lo requieran la organización y el mercado en el que actúa la firma. Se puede distinguir una flexibilidad pasiva u horaria de otra activa o funcional. La primera es característica de organizaciones híbridas que requieren que los trabajadores estén disponibles en horarios y bajo esquemas de
3 Las seis dimensiones consideradas son las siguientes: i) la existencia y complejidad que adquieren los equipos de trabajo; ii) la importancia otorgada al desarrollo de

competencias por parte de los trabajadores. iii)

la autonomía que los

trabajadores tienen para el desarrollo de sus tareas; iv) las

posibilidades que los trabajadores tienen de participar; v) la

flexibilidad para el desarrollo de las tareas; y vi) el tipo de control que se ejerce sobre las actividades

desarrolladas por los empleados. (Erbes et al, 2014). 
trabajo no siempre convencionales, y responde a tareas estandarizadas, donde los trabajadores pueden ser reemplazados en cualquier momento. La flexibilidad activa se encuentra en organizaciones formativas que no están dispuestas a prescindir del conocimiento, las habilidades y la experiencia individual de sus trabajadores, por lo cual se apoyan en las capacidades que los empleados poseen para autoorganizarse y gestionar su tiempo de trabajo. La organización taylorista, por su parte, tiene una rigidez que está dada por un esquema de trabajo con calificaciones y turnos preestablecidos. $\mathrm{Y}$ en cuanto al control, él se materializa en los dispositivos organizacionales desarrollados para garantizar el cumplimiento de los objetivos empresariales. En la empresa taylorista, el control es directo y supone la existencia de una jerarquía que ejerce coerción sobre el trabajador. En la organización formativa, en cambio, predomina el autocontrol y la evaluación por pares o "ajuste mutuo", en los términos de Mintzberg (1980). Y en cambio, la organización híbrida tiene un control jerárquico, pero no se basa en el poder de coerción sino en el establecimiento y el cumplimiento de objetivos.

\section{Acerca de la gestión del conocimiento en un grupo de PyMes de la cadena de los hidrocarburos en la Cuenca Neuquina}

Según la bibliografía relevada, la escasa literatura abocada a estudiar el proceso de la gestión del conocimiento en las PyMEs muestra que las mismas experimentan una serie de dificultades para gestionar el conocimiento de sus integrantes, exhibiendo con frecuencia una fuerte centralización del mismo en el dueño-gerente, y restricciones para beneficiarse del intercambio de conocimientos con el entorno. A la vez, es sabido que el conocimiento se ha ido consolidando como un factor clave en torno al cual las empresas construyen sus ventajas competitivas dinámicas. En el caso de la industria de hidrocarburos, fenómenos tales como el envejecimiento y la disminución de la cantidad del personal empleado, la intensidad tecnológica y de conocimiento del sector, la división del trabajo entre empresas en forma de "anillos" y las relaciones asimétricas de poder, la irrupción de las tecnologías para la explotación de hidrocarburos no convencionales, entre otros, incrementan la importancia de una gestión del conocimiento en todas las empresas del sector, y en las PyMEs en particular. En este contexto, la investigación se propuso identificar cómo se da el proceso de gestión de conocimiento en las PyMEs que operan en el segmento del upstream del sector de hidrocarburosen la Cuenca Neuquina. Las firmas que integran dicho segmento participan por sí mismas o más frecuentemente prestan servicios de apoyo a la exploración y explotación de petróleo y gas.

De dicho objetivo general se desprendieron los siguientes objetivos particulares: (i) teniendo en cuenta la influencia central del dueño-gerente en este tipo de empresas evidenciada en la bibliografía, se indagó sobre su rol en la gestión del conocimiento; (ii) considerando la manera en que la firma maneja su planta de personal, se investigó cómo las personas aprenden dentro de la organización, los esfuerzos que se llevan a cabo para fomentar su implicación en las tareas que realizan y aspectos relativos a la división del trabajo que puedan influir sobre la implicación y la autonomía de los recursos humanos; (iii) se caracterizó el proceso de gestión de información y conocimiento en cada una de las firmas indagadas, 
tanto a nivel interno como con respecto al intercambio de conocimiento con empresas e instituciones del entorno.

A los efectos de abordar esta temática en PyMes, siguiendo a Bamji et al. (2003), se asumió como hipótesis que la mayoría de este segmento de firmas desarrollan esfuerzos informales de mejora y no políticas explícitas. En cuanto a la división de las políticas de gestión del conocimiento entre aquellas que apuntan a codificarlo y las que no alteran su naturaleza explícita, se reconoció que aunque los procedimientos no estuvieran normados, las empresas pueden transferir, apropiar y retener el conocimiento de una forma exitosa.

Interrogantes planteados:

En función de los objetivos perseguidos en la investigación, se avanzó en el análisis de los casos de estudio sobre las siguientes preguntas-guía:

1. Sobre el papel del dueño-gerente: ¿En qué medida el conocimiento y la toma de decisiones estaban centralizados en el dueño-gerente al momento de la fundación de la empresa? ¿Cómo evolucionó esta situación según se fue modificando la planta de empleados? 2. Sobre la gestión de los recursos humanos: ¿Qué características reúne el personal que se incorpora a la organización? ¿Cómo se lleva a cabo la inducción del mismo? ¿Cómo es la división interna del trabajo? ¿Incluye esfuerzos para fomentar la implicación del personal? 3. Sobre la gestión de la información y el conocimiento: En cuanto al conocimiento interno, ¿En qué medida y de qué forma transforma la organización a los datos en información y conocimiento, y los utiliza para la toma de decisiones? ¿Cómo promueve la organización el aprendizaje individual y colectivo de sus miembros y cómo se dan las etapas de la gestión del conocimiento? ¿Qué capacidades dinámicas posee la firma? En cuanto a la relación con fuentes externas de conocimiento, ¿De qué forma y en qué medida la firma establece relaciones con las empresas e instituciones de su entorno para aprovechar las externalidades de conocimiento generadas? En función del análisis de la gestión interna y externa del conocimiento, ¿Cuál es la modalidad de aprendizaje adoptada por la empresa?

\section{Aspectos metodológicos}

\subsection{Estrategia de investigación}

Se desarrollaron ocho estudios de casos de acuerdo a lo que Yin (1984) denomina "estrategia de múltiples casos de estudio". Por la naturaleza de la investigación (descripción y explicación de la gestión de conocimiento, que por lo general se apoya en dinámicas que se caracterizan por la informalidad dificultado realizar mediciones), y dado el carácter eclético del enfoque conceptual adoptado, se optó por la metodología cualitativa.

La investigación se enmarcó en el Proyecto de Vinculación Tecnológica: "Fortalecimiento de las capacidades de generación de valor y gestión asociada del conocimiento y la inversión en PyMEs industriales y de servicios tecnológicos en el complejo de hidrocarburos en la Cuenca Neuquina. Acuerdo Universidad Nacional del Comahue y Plan Sustenta de YPF Regional Neuquén", aprobado mediante la 
Resolución 1793/14 de la Secretaría de Políticas Universitarias, con ejecución en 2015/2016.4

\subsection{Toma de información y selección de los casos objeto de estudio}

Siguiendo el marco teórico, a los efectos de realizar el trabajo de campo de la investigación, se diseñó una pauta guía de preguntas que incorporó los ejes conceptuales del mismo. Los ejes del cuestionario fueron: i) evolución y perfil actual de la empresa (actividad, escala, estructura, instalaciones, figura jurídica, conducción y organización, papel del dueño-gerente; inserción en el mercado, relaciones con clientes y proveedores.); ii) gestión del personal (modo de selección y contratación; capacitación; supervisión; procesos de toma de decisiones); iii) gestión de la información y el conocimiento interna y externa para la toma de decisiones (bases de datos de proveedores y clientes; planilla de control de procesos; planillas comparativas de precios; certificaciones de calidad y planillas de colada de cada partida; data mining sobre el balance productivo o de servicios en tiempos y costos; procesos de aprendizaje individuales y colectivos; innovaciones); iv) relación con el entorno: vínculos con instituciones científico tecnológicas; relaciones institucionales y con proveedores y clientes en la construcción de conocimiento).

Los casos trabajados integraron el grupo de empresas del Programa Sustenta de YPF de Desarrollo de Proveedores en la Cuenca Neuquina en 2015. En la indagación se recurrió a entrevistas semiestructuradas, realizadas a propietarios $y / 0$ gerentes técnicos 0 gerentes generales de las PyMEs, complementado ello con el material acerca de las firmas elaborado por técnicos del INTI y sus consultores en el marco del Programa. 5 Para la selección se adoptó el enfoque de “casos típicos" propuesto por Neergaard y Ulhøi (2007), según el cual los typical cases son aquellos que mejor reflejan el fenómeno a estudiar. A fin de que las firmas seleccionadas resultaran del subgrupo de casos típicos que resultan "reveladores", en términos de Yin (1984), con respecto al proceso objeto de estudio, y a partir de Neuman, et al, 2012, se adoptaron los siguientes criterios para seleccionar las unidades de análisis: que fueran empresas PyMEs con operaciones en la Cuenca Neuquina, manufactureras o prestadoras de servicios especiales, que evidenciaran el uso intensivo de conocimiento en algunos de sus procesos; que contaran con técnicos y/o profesionales en planta, indicador proxy de la cantidad de conocimiento disponible a gestionar, y que dispusieran de un laboratorio, departamento o sector de técnico en la empresa. Las 8 firmas del panel se repartieron entre el rubro metalmecánico: tornerías, fabricantes de tanques, de reparación de ductos, motores y componentes; el rubro electrónica, y dedicadas a servicios en yacimientos y talleres: calibración de válvulas, control de procesos, desparafinación y otros.
4 A partir de dicho

Proyecto surgieron trabajos que estudian el entramado empresarialinstitucional del sector $y$ los desafíos que implica la reconversión para la explotación de los hidrocarburos de reservorios no convencionales. Pueden citarse: Landriscini y Robles, 2016; Landriscini y Domeett, 2016;

Landriscini y Orlandini, 2015; Landriscini et al., 2015a; Landriscini,

2015a, 2015b.; y otros complementarios: Landriscini, Preiss y Robles, 2017;

Landriscini, Preiss, et al; 2016; Landriscini y Carignano, 2016.

5 El cuestionario utilizado consta en el Informe Final del Proyecto de Vinculación Tecnológica; Landriscini, Robles, Neuquén, abril de 2016. 
Luego de procesar las entrevistas y chequear la información obtenida de ellas con los técnicos del INTI Neuquén, con quienes se compartieron actividades en el marco del Proyecto, se elaboró una descripción de cada unidad relevada, estructurada de acuerdo a las temáticas abordadas por las preguntas semiestructuradas. Dicha descripción sirvió de insumo para el análisis realizado acerca de los casos, cuya síntesis se presenta en este documento, el que tomó como base las herramientas conceptuales que brinda el enfoque de theory building planteado por Eisenhardt y Graebner (2007). La información de las entrevistas a los casos seleccionados, se complementó con la información obtenida de otras firmas no integradas al Programa Sustenta, y relevadas como parte de la rutina de trabajo de campo en el contacto con las cámaras empresarias y con informantes calificados; de fuentes secundarias: estudios y proyectos y de la publicada en distintos medios periodísticos; y a través de la participación en foros y talleres sobre temáticas del sector de hidrocarburos.

\section{Análisis conjunto de los casos de estudio}

Efectuado el análisis de los casos individuales, el entrecruzamiento de los casos con ciertos conceptos teóricos permitió registrar una serie de interrelaciones valiosas para tomar en consideración en futuros abordajes de casos de PyMEs en general y aquellas del sector petróleo y gas, en particular. Se efectuó una clasificación en tres ejes temáticos tratados en los análisis de los casos: (i) el papel que asume el dueño-gerente o el equipo emprendedor; (ii) la gestión de los recursos humanos; y (iii) la gestión de la información y el conocimiento.

\subsection{Trayectoria empresarial y path-dependence de conocimiento}

En función de lo que en la teoría se conoce como path dependence (David, 2000), se puede observar que el origen de la empresa es un factor que condiciona en gran medida la evolución posterior de la delegación de la toma de decisiones y del conocimiento necesario para la toma de esas decisiones. Incluso se observan algunas situaciones en que se desaprovecha el conocimiento de los operarios, en tanto se les otorga menor intervención en el proceso decisional, de la que podrían asumir en función del conocimiento que poseen sobre el proceso productivo y sobre la relación con el cliente. Esta "dependencia del sendero" se tuvo en cuenta predominantemente en el caso de unidades que surgieron como desprendimientos de firmas en las que los titulares se desempeñaban como técnicos o profesionales. Tres de las empresas relevadas surgieron como desprendimiento de compañías multinacionales del primer anillo de proveedores. Sin embargo, luego siguieron trayectorias diferentes: una de ellas pasó a competir con la firma de la que se desprendió; otra se consolidó como proveedor de la EMN, y la tercera comenzó siéndolo, pero luego logró la “movilidad ascendente", pasando a integrar el primer anillo de proveedores. Otras dos firmas que se desprendieron del segundo anillo también mostraron diferencias: una de ellas permaneció en el segundo anillo; la otra pasó a integrar lo que sería un "tercer anillo" con una relación privilegiada con su empresa "madre". Los tres casos restantes, aparecían como prestadores de servicios altamente especializados; dos prestan servicios directamente a empresas 
del núcleo de la industria, mientras que el tercero lo hace de manera mediata, prestando servicios a empresas del primer anillo. De ello se deduce que: si bien no se verifica una tendencia clara que permita identificar unívocamente de qué manera influye el origen de las empresas en su posterior desenvolvimiento, se pueden extraer algunos aprendizajes a partir del análisis de las empresas que, siendo PyMEs, han podido consolidar su posición de "proveedores del primer anillo".

\subsection{Las PyMEs del "primer anillo"}

Cuatro de los casos estudiados pudieron consolidarse como proveedores del primer anillo, particularidad que reviste cierto interés si se tiene en cuenta que, por lo general, predominan en el primer anillo empresas multinacionales proveedoras de servicios especializados (Kozulj y Lugones, 2007; Landriscini, Robles, y Carignano, 2015b). Este grupo de empresas prestan servicios que tienen un alto grado de especialización, con profesionales o técnicos en planta. La capacidad de brindar un servicio altamente especializado con una estructura reducida resulta en un grado de flexibilidad superior al que muestran las empresas grandes que ofrecen servicios similares. Esta posibilidad se ha podido desarrollar gracias a una combinación entre el conocimiento que el dueño-gerente o el equipo emprendedor "importó" a la nueva unidad desde su experiencia de trabajo anterior, y la disponibilidad de recursos humanos formados o en formación.

\subsection{La delegación en el proceso decisional y la interdependencia del conocimiento}

En cuanto a las tipologías de conocimiento mencionadas por el informe de la OCDE (1996), se observa: por un lado, una interdependencia entre dichas tipologías, lo que se corresponde con la "indivisibilidad" del conocimiento que señala Antonelli (2003), y por otro lado, el hecho de que se produce una retroalimentación entre el conocimiento delegado a los individuos y su poder de decisión. Como apreciación general, en cuanto a las cuatro tipologías mencionadas por la OCDE se observa que: 1. El know-how ha sido distribuido en todos los casos a los empleados para el desarrollo de sus tareas. Sin embargo, la transferencia únicamente del know-how no es suficiente para lograr lo que Erbes, et al. (2014) Ilaman una "polivalencia enriquecedora", así como tampoco implica que la organización esté tomando medidas activas para promover la intervención del trabajador en el proceso productivo que desarrolla. Salvo en dos casos, se observa que en el resto, el knowhow de la firma proviene de la expertise desarrollada por el socio-gerente o el equipo emprendedor en su relación con empresas multinacionales del sector. En los dos casos señalados, el socio-gerente o equipo emprendedor contaba con knowhow relativo a la gestión de empresas y a la gestión comercial; el know-how técnico debió ser adquirido en el mercado laboral. 2. Con respecto al know-who, corresponde distinguir entre el know-who interno de la empresa, lo que autores como Edvardsson y Durst (2013a) llaman "knowledge map", y el know-who del entorno de la firma. En el caso de las empresas que tienen mandos medios ( 5 casos), son ellos quien detentan el knowledge map, el cual es utilizado para decidir a quién asignarle cada trabajo; en el resto de las empresas lo centraliza el sociogerente o el equipo emprendedor. Contrario a este tipo de conocimiento que es 
inherente a la firma, el know-who relativo al entorno de clientes, proveedores e instituciones, se obtuvo a partir de la experiencia previa en empresas del rubro en todos los casos, excepto en dos, en los que se debió desarrollar desde cero en base al know-how de gestión de tipo general de los fundadores. 3. El know-what como "información acerca de los hechos" (OCDE, 1996, p. 12), tiene en los casos analizados una relación importante con el know-who, si se considera que predomina la información tácita por sobre la codificada. Sólo dos casos hacen un importante uso de las TICs para codificar la información y reutilizar el conocimiento, igualmente la mayoría de las decisiones en estas empresas se toman mediante conocimiento informal obtenido de clientes y proveedores, en los que el know-who es esencial. 4. Así como el know-what se relaciona al know-who, el know-why proviene de una mezcla entre el conocimiento científico de los principios subyacentes a la tarea que se está realizando y lo que Davenport y Prusak (1998) llaman "ground truth", es decir, la comprobación empírica de ese conocimiento mediante su puesta en práctica. En todos los casos, salvo en uno, el personal profesional o técnico altamente calificado es quien concentra este tipo de conocimiento, en base al cual cinco de las ocho empresas han logrado generar innovaciones de producto, dos han logrado innovaciones de proceso, mientras que una de las firmas no logra fabricar sus propios productos.

El know-how relativo al proceso se distribuye en general entre todo el personal, mientras que el know-who y el know-what los concentran las personas que tratan con los clientes y con los proveedores y realizan esfuerzos de data mining; en los casos analizados son los mandos medios y/o los socios gerentes quienes concentran estos dos tipos de conocimiento. Sin embargo, es el know-why el tipo de conocimiento que ha permitido a dos de las empresas desarrollar sus propias piezas y patentarlas, a tres de ellas introducir adaptaciones en sus equipos y en dos de los casos el hecho de difundir ese know-why mediante capacitación in-house, les da mayor autonomía a los trabajadores para tomar decisiones y realizar sugerencias de innovaciones con relación a la tarea que desempeñan. Sin embargo, tal como coinciden en señalar tanto los teóricos de la escuela de la "innovación de conocimiento" (Nonaka et al., 2000; Nonaka, 1995; Nonaka, 1991) como los de la escuela del "aprendizaje organizacional" (Garvin et al., 2008), no alcanza con difundir el conocimiento, sino que el entorno organizacional debe ser propicio para la participación de los trabajadores, es decir que la comunicación debe ser "bidireccional" (Hatchuel et al., 2002).

\subsection{La gestión de recursos humanos}

En este punto resultó de interés analizar en qué medida las organizaciones aprovechan las externalidades relativas a la formación de la fuerza de trabajo, que produce el sistema de innovación en el que operan. También se consideró valioso conocer de qué manera la organización expande ese conocimiento favoreciendo una mayor autonomía del personal y la delegación del proceso decisional. En el conjunto de empresas seleccionadas, se observó que todas intentan incorporar como mínimo egresados de colegios secundarios técnicos, lo cual en algunos casos suplen con experiencia en el rubro; cuatro de las firmas también incorporan profesionales para integrar sus mandos medios e involucrarse en la prestación del 
servicio. En general, predominaron en los casos relevados esfuerzos informales de transferencia de conocimiento en los procesos de inducción de nuevo personal. Cuatro de las firmas evidenciaron intercambios más direccionados a las competencias que se pretende desarrollar para el puesto, mencionándose en tres de ellas la evaluación de desempeño y en dos, la rotación entre tareas de diferente nivel de dificultad.

Teniendo en cuenta los tres arquetipos que establecen Erbes et al. (2014), quedaron conformados tres subgrupos de empresas en función a la cercanía con respecto a alguno de esos arquetipos: dos de las firmas se correspondieron con el arquetipo taylorista; otorgan una reducida importancia a la formación de competencias, lo cual se retroalimenta negativamente con una limitada delegación del poder decisional. La polivalencia que se busca en estas organizaciones es pasiva, con lo cual una vez que los trabajadores están formados sus conocimientos no se regeneran, lo cual sería necesario para que los mismos mantengan su valor. Otras dos prestan servicios altamente especializados; sin embargo lo hacen mayormente a partir del conocimiento del dueño o equipo emprendedor. En este caso la comunicación llega a ser bidireccional por la proximidad del emprendedor con los operarios; sin embargo, la organización no realiza esfuerzos por continuar la formación del personal una vez que termina el período de inducción. Es por eso que no surgen innovaciones a partir de esa comunicación bidireccional, ni se otorga autonomía a los trabajadores. Estas empresas se aproximan al arquetipo híbrido. Las cuatro organizaciones restantes son formativas en el sentido que se enfocan en desarrollar las competencias del personal para poder delegarle progresivamente un mayor poder de decisión y una mayor autonomía en el desarrollo de sus tareas. En base a este mayor conocimiento, la comunicación bidireccional (Hatchuel et al., 2002) rinde sus frutos, materializándose en innovaciones de producto y/o de proceso y desarrollando profesionales o técnicos que generan nuevos conocimientos en base a la reflexión en la práctica (Schön, 1983).

\subsection{La gestión de la información y el conocimiento en la toma de decisiones (aprendizaje, capacidades dinámicas)}

Es sabido que la utilización de unidades de información o de conocimiento como inputs de los procesos productivos, pueden ayudar a disminuir la incertidumbre y a lograr una visión más aproximada del entorno organizacional que permita tomar decisiones con un sustento que exceda lo intuitivo. En este sentido, Grant (1996) reflexiona sobre la estructura organizacional que permitiría la toma de estas decisiones informadas, en el caso de una firma en que "la producción (y las decisiones sobre la producción) requieren varios tipos de conocimiento, [el cual] reside en numerosos individuos y los mecanismos de integración sólo involucran una proporción pequeña de los miembros" (Grant, 1996: 118). Agregado a ello, respecto de la integración de los conocimientos para la toma de decisiones, algunos teóricos ponen énfasis en la utilización de las TICs como medios para concretar dicha integración (Jaramillo Urrutia, 2007; Talisayon, 2006). Dixon (2000), en cambio, señala que no alcanza con el empleo de TICs, sino que éste debe complementarse con un entorno favorable al aprendizaje, y de diálogo bidireccional en la organización, y que estas tecnologías no reemplazan el contacto cara a cara, sino 
que sirven para complementarlo. Con relación a los casos estudiados: por un lado, las empresas relevadas no evidencian una planificación a largo plazo ni mecanismos complejos para la toma de decisiones; esto parcialmente puede explicarse por la volatilidad de la industria y la dependencia del nivel de actividad de las firmas con respecto al núcleo de operadores y a los subsiguientes anillos de proveedores de acuerdo a la posición que ocupen dentro de la industria; es probable que los factores que influyen para esta baja utilización de información para la toma de decisiones tengan que ver con las capacidades de la empresa y del equipo emprendedor, cuyos conocimientos están más ligados a lo productivo y lo relacional que a la gestión en la mayoría de los casos; por otro lado, se verifica un acceso diferencial a las fuentes de información consultadas para la toma de decisiones, que va en línea con el grado de delegación de dicho proceso en los empleados. En las organizaciones que sistematizan su conocimiento ya sea de manera tácita depositándolo en los mandos medios (tres casos), o bien codificándolo con apoyo de TICs (dos casos), esto representa un insumo para el proceso decisional, y a la vez representa cierta posibilidad de los operarios de influir en el proceso productivo mediante el conocimiento adquirido en la práctica. En las organizaciones que no producen conocimiento en sus empleados más allá de la inducción del personal ingresante (tres casos), el proceso decisional está centralizado en el equipo emprendedor o el sociogerente, quienes concentran el acceso al conocimiento utilizado para la toma de decisiones, y, por lo tanto, a las limitadas bases de datos que la firma maneja. En este punto es dable destacar la utilización por parte de dos de las firmas de sistemas informáticos diseñados a medida, para codificar parte del conocimiento relativo a las operaciones. Mientras que en un caso el acceso a dicho sistema es generalizado, en el otro, el mismo aún se hallaba en proceso de implementación.

\subsection{Aprendizaje, transferencia de conocimiento y rol de los mandos medios}

En cuanto a los procesos de aprendizaje individual y conjunto facilitados por el entorno de la organización, se observó que en los casos en que existe una menor delegación en el proceso decisional, prima un entorno de creación de conocimiento ("originating ba") al momento de la inducción de los trabajadores mediante la transferencia que Dixon denomina "en serie". ${ }^{6}$ Esta es la situación de tres de las firmas de la muestra, en las cuales, al nivel de los empleados, a posteriori de la capacitación del ingresante, no se le delega una mayor autonomía para intervenir en el proceso productivo, ni para "cuestionar sus supuestos", con lo que no terminan consolidándose en "organizaciones de doble bucle" en el sentido que plantea Argyris (1976). La situación del dueño-gerente o

\footnotetext{
6 "La transferencia en serie es un proceso que pone en movimiento el conocimiento peculiar que ha construido cada individuo que forma parte de un grupo o de un espacio público, de modo que pueda integrarse y ser comprendido por el equipo completo" (Dixon, 2000, p. 37)
} 
el equipo emprendedor en estas organizaciones es diferente, ya que ellos son los que centralizan el conocimiento de la firma en base a su comprensión integral del proceso y su interacción con el entorno. En las demás organizaciones, el "originating $b a "$ no se restringe al ingreso de nuevos empleados, sino que se prevén ciertos mecanismos tácitos (tres casos), complementados en los dos que usan las TICs con intensidad, con mecanismos de codificación, para la socialización de los conocimientos. En estas organizaciones juegan un papel central los mandos medios como centralizadores del conocimiento de los operarios, vehículos para la comunicación bidireccional, organizadores del proceso productivo y de los procesos de socialización del conocimiento de sectores. Estos casos complementan la transferencia de conocimiento "en serie" con otros tipos de transferencia: "lejana" en las cuatro firmas que prestan servicios en el yacimiento. El conocimiento adquirido en la reflexión durante la práctica se transfiere a otras áreas de la organización tales como la fabricación, en dos de ellas, o la reparación y mantenimiento de equipos, en las dos restantes. Y "cercana" en dos de los casos, en los que se codifica el conocimiento con miras a su reutilización. Los resultados que muestran las empresas que delegan parte de la toma de decisiones en sus mandos medios son satisfactorios en la medida que promueven intercambios de conocimientos de los mandos medios entre sí, como también entre ellos y los socios-gerentes o el equipo emprendedor. En ese sentido, los equipos emprendedores también permiten un intercambio de conocimiento que no ocurre internamente en una firma unipersonal (Kantis et al., 2004, 2011).

\subsection{Los vínculos con clientes y la adaptación de tecnologías como capacidades}

Al analizar las capacidades con que cuentan las PyMEs estudiadas para producir resultados innovadores, puede afirmarse, como primera aproximación, que la vinculación más importante que se visualizó con el entorno es la relación user-producer (Lundvall, 1985); los requerimientos del cliente, impulsan a las firmas a desarrollar comportamientos innovativos en sentido amplio. Con frecuencia, las respuestas adaptativas de las firmas a los cambios en los requerimientos de sus clientes tienen que ver con esfuerzos innovativos incrementales y no disruptivos, con lo cual entran en la definición de innovación que plantea el Manual de Oslo de la OCDE (1997), que incluye tanto innovaciones de producto, como de proceso, nuevas para la firma, y no sólo aquellas innovaciones socialmente nuevas. Así, las capacidades de vinculación con sus clientes y proveedores son complementadas por las firmas con una capacidad de producción en las que las actividades de investigación y desarrollo se vinculan más con los esfuerzos de asimilación, adaptación y mejora de tecnología importada que con una etapa de capacidad innovativa per se. Existe, además, una retroalimentación entre la prestación del servicio o la fabricación del producto con un criterio de calidad para la construcción de una reputación que les permite desarrollar su capacidad de vinculación. Más allá de lo general, algunos casos merecen ser mencionados aparte: una firma ha logrado en base a la sinergia entre capacidad de producción y de vinculación una "movilidad ascendente" entre anillos, mientras que en otras dos la vinculación con el cliente les ha permitido adaptar sus productos a las condiciones requeridas, fortaleciendo la capacidad de vinculación y la de producción. 
Con respecto a la capacidad de inversión, la reinversión de utilidades es la principal o única fuente de inversión en la mayoría de las firmas. Solo dos utilizan instrumentos de financiamiento bancario o fondos públicos. La relación asimétrica con las empresas a las que proveen introduce incertidumbre en sus flujos de fondos futuros, lo cual impacta en la disponibilidad de capital para reinvertir, y en el acceso a financiamiento externo.

\subsection{Grado de aprovechamiento de las fuentes de conocimiento externo}

El conjunto de empresas relevadas mostró una modalidad de aprendizaje "restringida" en los términos de Robert (2012), la cual "se caracteriza por estar centralizada predominantemente en las fuentes internas y tácitas del conocimiento". (Robert, 2012: 29). Ninguna explota adecuadamente el conocimiento externo en base a interacciones formales basadas en transacciones de conocimiento (modalidad corporativa), o una predominancia de saberes tácitos intercambiados mediante la experimentación inter-firma (modalidad distribuida) (Antonelli y Barbiellini Amidei, 2011). Si bien todas las firmas entrevistadas se relacionan con sus clientes y sus proveedores, lo cual en general posibilita su supervivencia y crecimiento, e introducen ciertos cambios en sus productos o servicios en base a los requerimientos de sus clientes, sólo dos de ellas, señalaron recibir capacitación de sus proveedores. Es decir que las relaciones con empresas del entorno están ligadas casi exclusivamente a las transacciones. Una sola firma dio cuenta de mantener relaciones de intercambio técnico con su proveedor de cabinas de control geológico, que pertenece a su mismo grupo económico. Por el lado de las organizaciones públicas de investigación, en los términos de Dutrénit et al. (2010) el relacionamiento se da por los canales: (i) tradicional, mediante la contratación de profesionales de las universidades de la zona, o bien de escuelas técnicas; y (ii) de servicios, en tanto algunas de ellas se han relacionado puntualmente requiriendo los servicios de INVAP (un caso), INTI (cuatro casos), e IRAM (un caso).

Tal como señalan Cohen y Levinthal (1990), la absorción de conocimiento del entorno está condicionada al desarrollo de ciertas capacidades y una base de conocimiento que permita a la firma esa absorción. En las organizaciones en las que el conocimiento se halla predominantemente concentrado en el equipo emprendedor o en el socio-gerente, son ellos quienes toman el conocimiento externo para incorporarlo a la firma. En los casos que cuentan con mandos medios (cuatro), ellos también colaboran en la incorporación de conocimiento del entorno a la vez que sirven de repositorio de los saberes internos.

\section{Resultados}

De la investigación realizada se derivan una serie de implicancias para las PyMEs del sector relacionadas a posibilidades de aprendizaje a partir de los casos exitosos y de la bibliografía consultada. Estas potencialidades se agrupan en las relacionadas a la gestión interna, y las vinculadas a la gestión externa.

a) Relacionadas a la gestión interna: Los casos relevados muestran que la alta rentabilidad que experimentó la industria entre 2003 y 2014, con un intervalo de inestabilidad en el año 2008, complementada con la reactivación que siguió a la 
renacionalización de YPF, permitió a las PyMEs del complejo hidrocarburífero regional sobrevivir, e incluso crecer, a pesar de las asimetrías estructurales que rigen en la cadena y de adolescer de ciertas ineficiencias. Éstas en general no se limitan a la baja productividad operativa, sino que responden a una insuficiente generación de información para la toma de decisiones que se corresponde con un proceso decisional poco complejo, una escasa delegación de responsabilidades y transferencia del conocimiento, en la cual en varios de los casos se limitan a transferir el know-how esencial para la prestación del servicio, centralizando el resto del conocimiento el dueño-gerente o el equipo emprendedor. Si en un contexto de crecimiento, la gestión de los conocimientos era útil por su potencialidad de aumentar la rentabilidad, en un contexto de caída del sector se vuelve necesaria para aumentar la productividad, mejorar la ecuación de costos y permitir la supervivencia de las firmas en el sector.

La centralidad del papel del dueño-gerente o el equipo emprendedor no es un fenómeno extraño en una PyME. En los casos estudiados los fundadores parten de la base de su experiencia en empresas multinacionales del primer anillo de proveedores, contando con experiencia en uno de los servicios especiales que demanda la industria, y se limitan a crear una empresa con foco en desarrollar ese único proceso de la mejor manera posible. Con la excepción de tres de los casos, la expertise de negocios escasea en las empresas relevadas, evidenciándose esto, por ejemplo, en que ninguno de los casos relevados muestra experiencias de diversificación relacionada o no relacionada. Las firmas relevadas, en las que el poder de decisión aparece como más concentrado en el dueño-gerente o en el equipo emprendedor, experimentan una retroalimentación entre la concentración del poder de decisión, del relacionamiento con el entorno, y, por lo tanto, del aprendizaje proveniente de esas dos fuentes. En estos casos, con la inducción del personal concluye su formación, con lo cual luego la comunicación dentro de la organización pasa a ser unidireccional. Otros casos exhiben un conocimiento que está más distribuido - de manera tácita o codificada; en tales casos el rol de los mandos medios resulta clave.

Pareciera deducirse de las investigaciones de Kozulj y Lugones (2007) y de Landriscini et al. (2015a) que el hecho de ubicarse en un "anillo de proveedores" más cercano al núcleo operativo puede ser favorable a las condiciones contractuales de la relación de las empresas con sus clientes. En este sentido, un subgrupo de la muestra seleccionada ha logrado consolidar su posición en el primer anillo de proveedores. Cuatro firmas prestan servicios de elevada complejidad con una flexibilidad superior a sus competidores de mayor escala de negocios, por su carácter de PyMEs. Una firma, por su parte, integra el "tercer anillo" de proveedores, con su know-why tercerizado, y una capacidad financiera muy limitada que es parcialmente responsable por su reducido dinamismo.

El papel de los mandos medios -en las firmas que los poseen- es esencial, teniendo en cuenta que: i) su presencia permite intercambios de conocimiento a un cierto nivel de agregación y entre sectores de la firma que no se dan en el caso de empresas en las que hay un dueño-gerente que se relaciona directamente con los operarios (dos casos de la muestra); ii) ellos son quienes aglutinan el conocimiento que el resto del personal operativo adquiere en la prestación de las tareas, y combinando ese know-who interno de la organización con el know-why en sus 
reflexiones junto con el dueño-gerente o equipo emprendedor han posibilitado innovaciones de producto o de proceso; iii) El know-why en los casos que cuentan con personal profesional en su dotación, no sólo ha permitido innovaciones de producto o de proceso sino que le permite a la organización reconocer el conocimiento que se produce internamente para socializarlo al resto de los empleados y poder reutilizarlo. Las organizaciones que no pueden hacer eso, dependen del conocimiento que obtienen del entorno, recurso que no está demasiado explotado en el conjunto de firmas analizado.

Siguiendo la línea argumentativa del feedback entre delegación de responsabilidades y transmisión de conocimiento, las organizaciones cuyo proceso formativo finaliza con la inducción del personal muestran a su vez un empobrecimiento del trabajo como sistema de actividad. Incluso en las organizaciones relevadas que se identificaron como híbridas, no se posee una visión de la necesidad de que todos los niveles de la organización aporten ideas que representen mejoras en el proceso productivo. De esta manera, la organización omite reconocer el conocimiento que podrían aportar los trabajadores al diseño más eficiente del proceso en base al desempeño del mismo, generándose un entorno que sólo favorece el diálogo unidireccional top-down. Además, en estos casos en que el conocimiento y la facultad decisional está concentrada en el dueñogerente, la organización cuenta con límites al crecimiento dados por su capacidad de gestión. El contraejemplo de esto serían empresas que en la muestra cuentan con cierto know-how de negocios, con lo cual valoran en mayor medida el input que puedan brindar los trabajadores. Uno de los perjuicios del empobrecimiento del trabajo, constituye la migración de los trabajadores hacia las empresas trasnacionales más grandes del sector. Si bien la autonomía que se brinde a los trabajadores debe ir acompañada del conocimiento que se corresponde con cada nivel, el análisis de casos evidencia que los beneficios de la transferencia de conocimiento pueden materializarse en la medida en que la empresa forma de competencias particulares en sus empleados, de acuerdo a las necesarias para cada puesto, combinando ese enfoque con la rotación entre tareas de diferente dificultad y una acorde evaluación de desempeño periódica. Esto coincide con algunas características de las organizaciones formativas que mencionan Erbes et al. (2014).

La utilización de tecnologías de la información y el seguimiento de indicadores y utilización de bases de datos como apoyo a la toma de decisiones ha sido por lo general baja entre las firmas relavadas. Esto se corresponde con una planificación limitada a las cuestiones operativas de corto y mediano plazo a la que se suman algunas decisiones relativas a la inversión. Ninguna de dichas empresas utiliza herramientas para el seguimiento periódico de indicadores relevantes, tales como el Tablero de Comandos de Kaplan y Norton (1992), ni intentan mitigar la volatilidad de sus fuentes de ingresos mediante la planificación por escenarios a mediano-largo plazo.

En cuanto a la circulación de conocimiento al interior de la organización, tanto los mecanismos de socialización del conocimiento tácito como los de externalización de conocimiento codificado han mostrado en los casos estudiados resultados relevantes en términos de innovación de productos y de procesos. Mientras que para quienes codifican el conocimiento es importante la transferencia 
"cercana", para las firmas que operan en el yacimiento resulta valioso introducir adaptaciones en sus productos o procesos en base a lo que aprenden sus empleados a campo, o sea valoran la transferencia "lejana".

Las firmas que han registrado un menor dinamismo y menores esfuerzos innovativos han sido aquellas en las que el fomento al aprendizaje se interrumpe luego de la inducción del personal ingresante, y aquellas en las que predomina la comunicación unidireccional top-down por una excesiva concentración del conocimiento en los dueños o gerentes. Como fuera mencionado, la delegación de parte de la toma de decisiones y del conocimiento en los mandos medios los consolida como repositorios de conocimiento para el resto de la organización y poseedores de un knowledge-map, o “know-who interno". Esto les permite reconocer los avances de conocimiento interno invidual a fin de propiciar formas de socializarlo o externalizarlo. Cabe señalar que independientemente de que la transferencia de conocimiento interno se realice en su estado tácito o se codifique, el carácter bidireccional de la comunicación ha exhibido beneficios.

La capacidad central en estas firmas es la vinculada a la producción con calidad y flexibilidad, dado que su captación de clientes se produce predominantemente en base a su reputación. Complementan su capacidad de producción la habilidad de asimilación, adaptación y mejora de equipos importados, con una ausencia de un criterio de mejora continua. Constituye para ellas un desafío acceder a instrumentos de financiación externa (salvo dos excepciones), lo cual impide el escalamiento de sus actividades si se considera en conjunto con la inestabilidad de los flujos de ingresos, derivada de la posición que cada empresa ocupa en la industria. Mientras que el acceso externo puede ser atribuído en parte a la opacidad de la información producida por las PyMEs, y, en parte, a la oferta inadecuada existente en el entorno, la mayor estabilidad de los flujos de fondos para su reinversión debería provenir de una diversificación, una mejora en las condiciones de la contratación, o al menos una disminución de la incertidumbe a partir de la formacilización de los procesos de planificación y toma de decisiones.

b) Las fuentes de conocimiento externas. La vinculación con el entorno empresarial-institucional de las firmas estudiadas mostró una modalidad de aprendizaje restringida, en la que el crecimiento y el aprendizaje colectivo se ve fuertemente demorado por el escaso aprovechamiento de las relaciones con el entorno para apropiarse de nuevos conocimientos. Con respecto a la relación con otras empresas, los casos estudiados registran algunos avances en base a su relación transaccional con sus clientes. Los requerimiento especiales de ellos exigen esfuerzos de data mining para conocer si la empresa puede brindar ese servicio u ofrecer ese producto y en qué condiciones lo puede hacer. La ausencia de proyectos colaborativos con otras empresas a nivel horizontal o vertical es el denominador común en las experiencias estudiadas, lo cual podría acercarlas a lo que Antonelli y Barbiellini Amidei (2011) denominan una "modalidad distribuida" de experimentación y aprendizaje interfirma. En los tres casos estudiados que evidenciaron una menor distribución de la toma de decisiones al interior de la firma, esta centralización coincidió con una concentración de la facultad de relacionamiento con el exterior en el dueño-gerente o el equipo emprendedor. Estas unidades surgen con base en la experiencia de los emprendedores en empresas multinacionales, y ellos utilizan esa base de conocimientos para seguir 
aprendiendo del entorno. Para complementar la afirmación de Rojo Brizuela et al (2011) con respecto a la primacía de la transferencia de conocimiento desde las empresas nacionales a las multinacionales por sus trabajadores, se puede afirmar que en este caso los emprendimientos spin off surgen utilizando como base el conocimiento que los emprendedores adquieren en su experiencia laboral, muchas veces en empresas multinacionales del sector.

En la misma línea, las relaciones con las instituciones de conocimiento del sector se restringen al canal tradicional, específicamente a la contratación de personal egresado de escuelas técnicas o universidades, y al canal de servicios en oportunidades puntuales. En este sentido la potencialidad del desarrollo de un canal bidireccional de relaciones a largo plazo con instituciones de conocimiento tales como universidades, entre otras, está lejos de materializarse.

\section{Reflexiones y aspectos a considerar en una política PYME}

En función de la consolidación de los servicios de mayor complejidad asociados a la exploración y explotación de reservorios no convencionales, y al sistema técnico de la fractura hidráulica en la Cuenca Neuquina, y, por lo tanto, una ubicación de las empresas en un anillo de proveedores más próximo al núcleo, los policy makers deben evaluar las experiencias de PyMEs que han logrado posicionarse como proveedores de las empresas operadoras de la industria. El fortalecimiento de la capacidad de inversión de las firmas del sector depende tanto de la generación de instrumentos adecuados a la condición PyME para favorecer el acceso a capital externo, complementados con una mayor presencia de sociedades de garantía recíproca, como también de la generación de flujos de ingresos más estables de estas empresas. Una mejora en el posicionamiento de las PyMEs en la industria puede coadyuvar a la estabilidad de los flujos de fondos que perciben. Si bien los esfuerzos para lograr este upgrading deben provenir de las propias empresas, los responsables de las políticas en la materia podrían favorecerlo mediante la generación de externalidades de conocimiento de mayor cuantía y calidad, que las PyMEs puedan apropiar a fin de prestar servicios especializados. Estas externalidades deberían incluir informes sectoriales, estadísticas, estudios cualitativos, así como también una mejora del perfil de los egresados de escuelas técnicas y universidades. Para que la estrategia de diversificación de las fuentes de ingresos tenga mejores resultados, además de fomentar el upgrading de las empresas y su diversificación de productos, también corresponde analizar la estrategia de diversificación hacia mercados de la región con producción de petróleo y gas. Esto podría lograrse mediante la creación de un consorcio de exportación que produzca información de mercado y concrete acuerdos de negocio.

Cabe consignar asimismo el limitado nivel de desarrollo del Sistema Regional de Innovación en el que operan las firmas. Ello genera que sólo algunas de ellas puedan aprovechar las externalidades que en él se producen, para la certificación de calidad o la mejora de procesos a través del "canal de servicios". Las grandes de la cadena utilizan canales internacionales. Utilizando como benchmark el caso de Brasil, se observa que en el entramado de proveeddoras a los hidrocarburos, por ejemplo, se cuenta con la intervención activa de instituciones de investigación, 
apoyo y capacitación, universidades, el servicio de educación superior y demás servicios del "Sistema S" brasileño de aprendizaje para la industria y el comercio, el SEBRAE, de una escala incomparable con el Centro PyME ADENeu. Asimismo, atendiendo a la baja utilización de TICs para la gestión del conocimiento en la muestra estudiada, resultaría relevante la incorporación de la informática gratuita y de código libre para apoyar la gestión del conocimiento de las PyMEs.

En este sentido, corresponde destacar que las PyMEs que se ubican en el primer anillo enfrentan una competencia intensa por parte de empresas multinacionales de servicios especializados, que en la generalidad de los casos cuentan con externalidades de calidad en sus sistemas de innovación de origen que les permiten alcanzar altos estándares de calidad en la prestación de sus servicios.

La experiencia de este estudio muestra que las PyMEs que logran establecerse en el primer anillo lo hacen en base a la flexibilidad y capacidad de respuesta inmediata, adaptación de sus soluciones a las necesidades del cliente y a las características de los yacimientos, así como también mediante la adopción de estándares de operación que atestiguan la calidad de sus servicios.

Un programa de capacitación horizontal para la industria en materias de capacidades de gestión y capacitación permitiría a los empresarios reconocer los beneficios potenciales de una complejización del proceso decisional, lo cual complementaría los programas de proveedores de las operadoras. Esto también requiere la ampliación de la oferta de consultoría en business intelligence.

Finalmente, el nivel de interacción entre PyMEs es muy bajo, sumado a que existen varias cámaras empresarias. La creación de proyectos consorciados entre ellas podrían favorecer la transferencia de conocimiento interfirma, así como la cooperación para lograr mejores posibilidades de negociación con las grandes empresas; la conformación de consorcios entre PyMEs, grandes empresas e instituciones de conocimiento constituirían la mejor alternativa. De esta forma, quizás surgirían experiencias de vinculación por el "canal bidireccional" entre empresas e instituciones de conocimiento.

\section{BIBLIOGRAFÍA}

Aggio, C. et al; (2016) Desafíos y oportunidades de innovación en la producción de petróleo y gas no convencionales en la Argentina. Documento de trabajo $\mathrm{N}^{\circ} 10$. Centro Interdisciplinario en Ciencia Tecnología e Innovación. Buenos Aires. MINCYT.

Barletta, F. et al; (2016) Construcción de capacidades en las firmas argentinas. Más allá de los laboratorios de I-D. Revista PyMes, Innovación y Desarrollo, Vol. 4, Nº 3.

Barletta, F., et al (2014). Introducción. En Tópicos de la teoría evolucionista neoschumpeteriana de la innovación y el cambio tecnológico (pps. 11-32). UNGS: Buenos Aires: Miño y Dávila.

Becattini, G. (2002). Del distrito industrial marshalliano a la " teoría del distrito"

CEPAL. (2015). Impacto socioeconómico de YPF desde su renacionalización (Ley 26.741) Desempeño productivo e implicancias sobre los mercados laborales y el entramado de proveedores Vol. I. y Vol. II Santiago de Chile. 
Damaskopoulos, T. et al (2008). "Extended and Dynamic Clustering of SMEs". Engineering Economics, 56(1), 1-5.

Di Sbroiavacca, N. (2013). Shale Oil y Shale Gas en Argentina. Estado de situación y prospectiva. IIEE. Fundación Bariloche

Dini, M., \& Stumpo, G. (2011). Políticas para la innovación en las pequeñas y medianas empresas en América Latina. Documento de Proyecto. CEPAL. Santiago de Chile.

Easterby-Smith, M., y Prieto, I. (2007). "Dynamic capabilities and knowledge management: An integrative role for learning?" British Journal of Management, 19(3), 235-249.

Edvardsson, I. R., y Durst, S. (2013a). Does Knowledge Management Deliver the Goods in SMEs? Business and Management Research, 2(2), 52-60.

Edvardsson, I. R., y Durst, S. (2013b). The Benefits of Knowledge Management in Small and Medium-sized Enterprises. Procedia - Social and Behavioral Sciences, 81, 351-354.

Eisenhardt, K. M. (1989). Building Theories from Case Study Research. Academy of Management Review, 14(4), 532-550.

Eisenhardt, K. M., y Graebner, M. E. (2007). Theory building from cases: Opportunities and challenges. Academy of Management Journal, 50(1),

Engeström, Y. (2001). El aprendizaje expansivo en el trabajo: hacia una reconceptualizacion teórica de la actividad. Journal of Education and Work, 14 ( $\left.\mathrm{n}^{\circ}{ }^{\circ}\right)$, $1-16$.

Erbes, A., et al. (2014). El rol de la organización del trabajo en el desarrollo de procesos de aprendizaje. En Tópicos de la teoría evolucionista neoschumpeteriana de la innovación y el camio tecnológico (pp. 287-318). UNGS. Buenos Aires: Miño y Dávila.

Erbes, A. y Suárez, D. (Comps.) (2016) Repensando el desarrollo latinoamericano. Una discusión desde los sistemas de innovación. Ed. UNGS. Los Polvorines. Universidad Nacional de General Sarmiento.

Estrada, S., y Dutrénit, G. (2007). Gestión del conocimiento en PyMEs y desempeño competitivo. Engevista, 9 (dezembro), 129-148.

Evangelista, P., et al (2010). The Adoption of Knowledge Management Systems in Small Firms. Electronic Journal of Knowledge Management Volume, 8(1),

Ferraro, C., y Stumpo, G. (2010). Políticas de apoyo a las pymes en América Latina: Entre avances innovadores y desafíos institucionales. Santiago de Chile: CEPAL.

Grant, K. A. (2007). Tacit Knowledge Revisited - We Can Still Learn from Polanyi. Electronic Journal of Knowledge Management, 5(2), 173-180. 
Gestión del conocimiento y desafíos en PyMEs proveedoras de la cadena de hidrocarburos en la Cuenca Neuquina. Avance de investigación y reflexión para el diseño de políticas

Grant, R. M. (1996). Toward a knoweldge-based theory of the firm. Strategic Management Journal, 17(Winter Special Issue), 109-122.

Hatchuel, A.,et al. (2002). De la gestión de los conocimientos a las organizaciones orientadas a la concepción. Revista Internacional de Ciencias Sociales, (171), 29-47.

Kantis, H., et al (2004). Desarrollo emprendedor: América Latina y la experiencia internacional. G. Giannoni, Ed.. New York: BID.

Kantis, H., et al (2012). Políticas de Fomento al Emprendimiento Dinámico en América Latina: Tendencias y Desafíos (No. 09). Caracas.

Kantis, H., et al (2011). "Radiografía de las nuevas empresas de rápido crecimiento en la Argentina". Boletín Informativo Techint, (335), 77-92.

Kaplan, R. S., y Norton, D. P. (1992). "The Balanced Scorecard - Measures That Drive Performance". Harvard Business Review, 70(1), 71-79.

Katz, J., y Zamorano, F. (2011). Capacidad innovadora de las pymes y las políticas públicas : el caso chileno. Santiago de Chile.

Kozulj, R. (2011). Los principales desafíos del sector energético argentino: un panorama complejo (pp. 1-43). Neuquén: FAEA - Fundación Bariloche.

Kozulj, R., y Lugones, M. (2007). "Estudio de la trama de la industria de los hidrocarburos en la Provincia de Neuquén". En M. Delfini et. al. (comp.) - Innovación y empleo en tramas productivas de Argentina. UNGS. Los Polvorines. Ed.Prometeo.

Lall, S. (1992). Technological capabilities and industrialization. World Development, 20(2), 186.

Landriscini, S. G. (2015). "PYMEs de servicios petroleros en la Cuenca Neuquina. Trayectorias, políticas, instituciones y desafíos". En Lenguaje Claro (Ed.), V Jornada de la Industria y los Servicios -Buenos Aires. FCE UBA.

Landriscini, S. G., y Orlandini, M. M. (2014). "Innovación y coordinación en PyMEs de servicios petroleros en el cluster shale de Vaca Muerta". En Ascúa, R. et al. - Lecturas seleccionadas de la XIX Reunión Anual de la Red PYME Mercosur. Vol 1. pp. 230-299). Campinas: Instituto de Economía de la Universidad Estadual de Campinas..

Landriscini, S. G.., y Orlandini, M. M. (2015). "El desarrollo regional en la cuenca neuquina. Hidrocarburos no convencionales y múltiples desafíos". Revista Pilquén Ciencias Sociales, 18 (1), 50-62. CURZA. U.Nacional del Comahue.

Landriscini, S. G., et al (2015). "El escenario Shale y los desafíos en la Cuenca Neuquina. Aprendizaje tecnológico y creación de capacidades en PyMes proveedoras de servicios especializados a los hidrocarburos" En R. Ascua et al; Lecturas seleccionadas de la XX Reunión Anual de la Red PyME Mercosur. Bahía Blanca: UNS.UPSO.IIES:CONICET. Red PyME Mercosur. 
Landriscini, S. G. y Carignano, A. (2016) Subcontratación y tercerización en tiempos de volatilidad de los precios internacionales del petróleo. El procedimiento preventivo de crisis y sus derivaciones en las relaciones interempresarias y laborales en la Cuenca Neuquina VIII Congreso ALAST. Buenos Aires, 3 al 5 de agosto.

Lee, M. R., y Lan, Y. C. (2011). Toward a unified knowledge management model for SMEs. Expert Systems with Applications, 38(1), 729-735.

Link, A. N., y Antonelli, C. (2013). Building the Economics of Knowledge A Roadmap, Lundvall, B.-Å. (1985). Product innovation and user-producer interaction. Research Series (Vol. 31). Aalborg: Aalborg Universitetsforlag.

Lundvall, B.-Å. (2005). "National Innovation Systems - Analytical Concept and Development Tool". En National Innovation Systems - Analytical concept and development tool (p. 43). Copenhagen.

Marín, A. et al (2017) Recursos naturales y desarrollo productivo y tecnológico. Revista Desarrollo Económico. Vol. 56 n²20, ene-abril. pp. 469-470. Buenos Alres. IDES.

Marin, A., et al (2009), The possible dynamic role of natural resource-based networks in Latin American development strategies, Documento de trabajo, Buenos Aires, Cepal-segib.

Marzo Carpio, M. (2015) El desplome 2014-2015 de los precios del crudo: causas y previsiones a corto plazo. Fundación para la Sostenibilidad Energética y Ambiental. febrero. Barcelona.

Nelson, R. R., y Winter, S. G. (1982). An evolutionary theory of economic change. Cambridge MA Belknap (Vol. 93). Cambridge: Harvard University Press.

Neuman, M., et al (2012). Plan Estratégico para el Desarrollo de Proveedores de Bienes y Servicios de la Industria del Gas y del Petróleo. UNGS. CABA.

Nonaka, I. (1991). The Knowledge-Creating Company. Harvard Business Review, (Nov.-dec.),

Nonaka, I.,y\& Takeuchi, H. (1995). Teoría de la creación del conocimiento organizacional. En La organización creadora de conocimiento (pp. 61-103). Madrid: Universidad Iberoamericana.

OCDE. (1996). The Knowledge-Based Economy (Vol. 96). Paris.

OCDE. (1997). The measurement of scientific and technological activities - Proposed guidelines for collecting and interpreting technological innovation data - Oslo Manual (Vol. Third edit). Oslo.

OECD. (2001). Competencies for the knowledge economy. En Education Policy Analysis 2001 (p. 152). Paris: 
OECD, WTO, y World Bank Group. (2014). Global Value Chains: Challenges, opportunities and implications for policy. Report prepared for submission to the $\mathrm{G}_{20}$ Trade Ministers Meeting Sydney, Australia. Sydney.

Pérez, C. (2016) Teoría y política de desarrollo como blanco móvil. En Erbes, A. y Suárez, D. Repensando el desarrollo latinoamericano. una discusión desde los sistemas de innovación. Ed. UNGS. Los Polvorines.

Pietrobelli, C., y Rabellotti, R. (2005). Upgrading in Clusters and Value Chains in Latin America The Role of Policies. Washington, D. C.

Schumpeter, J. A. (1947). The creative response in economic history. The Journal of Economic History, 7(2), 149-159.

Segarra, M. (2006). Estudio de la naturaleza estratégica del conocimiento y las capacidades de gestión del conocimiento. Universidad Jaume I. Castellón. España.

Teece, D., y Pisano, G. (1994). "The dynamic capabilities of firms: an introduction". Industrial and Corporate Change, 3(3), 537-556.

Torres, O. (2004). "The SME concept of Pierre-André Julien: an analysis in terms of proximity". Piccola Impresa/Small Business, 2, 1-12.

Yin, R. K. (1984). Case Study Research: design and methods (No. 5). Applied Social Research Methods (Vol. 2).

Yoguel, G. et al; (2013) De Schumpeter a los postschumpeterianos: viejas y nuevas dimensiones analíticas. Problemas del desarrollo. Revista latinoamericana de economía. Vol 44. № 174. Julio-septiembre. pp. 35-59. Universidad Autónoma de México. Distrito Federal. México.

Zhou, H., y Uhlaner, L. M. (2010). Knowledge Management as a Strategic Tool to Foster Innovativeness of Smes. SSRN Working Paper Series.

Susana Graciela Landriscini. Departamento de Economia Facultad de Economia y Adminsitracion e Instituto de Estudios de Humanidades y Ciencias Sociales Uncomahue CONICET. Universidad Nacional del Comahue glandriscini@gmail.com

Lisandro Valentin Robles. Estudiante de Postgrado en Temas de Comercio Internacional. Universidad Nacional del Comahue. lilo.nqn@gmail.com 